\title{
Continuous Transition of Deformation Modes in Fe-30Mn-5Si-1Al Alloy*1
}

\author{
Motomichi Koyama ${ }^{1, * 2}$, Takahiro Sawaguchi ${ }^{2}$, Kazuyuki Ogawa ${ }^{2}$, \\ Takehiko Kikuchi $^{2}$ and Masato Murakami ${ }^{1}$ \\ ${ }^{1}$ Shibaura Institute of Technology, 3-7-5, Toyosu, Kouto, Tokyo 135-8548, Japan \\ ${ }^{2}$ National Institute for Materials Science, Tsukuba 305-0047, Japan
}

\begin{abstract}
Deformation modes at various stages of plastic deformation have been investigated at the same location in an Fe-30Mn-5Si-1 Al (mass\%) alloy specimen, which exhibits a good shape memory effect associated with the $\operatorname{FCC}(\gamma) \rightarrow \operatorname{HCP}(\varepsilon)$ martensitic transformation and a relatively high ductility caused by deformation twinning. The surface relief caused by the $\gamma \rightarrow \varepsilon$ martensitic transformation, deformation twinning and slip band formation has been analyzed by measuring the surface tilt angles corresponding to each deformation mode by atomic force microscopy. Although the $\varepsilon$-martensitic transformation is the dominant deformation mode at an early deformation stage, a part of the $\varepsilon$ martensite plates changes to deformation twins with increasing tensile strain. Slip deformation also occurs inside the same region under excessive strain. A continuous transition of these deformation modes occurs in other grains as well in the same order: $\varepsilon$ martensite $\rightarrow$ deformation twins $\rightarrow$ slip bands. [doi:10.2320/matertrans.M2010073]
\end{abstract}

(Received February 26, 2010; Accepted April 21, 2010; Published June 2, 2010)

Keywords: Twinning induced plasticity, shape memory, stress induced martensitic transformation, twinning deformation, slip deformation, atomic force microscope

\section{Introduction}

Some high manganese steels have been known to show a shape memory effect ${ }^{1-3}$ ) or a TWIP (Twinning Induced Plasticity) effect. ${ }^{4,5)}$ The shape memory effect is attributed to the $\varepsilon$-martensitic transformation ( $\mathrm{FCC} \rightarrow \mathrm{HCP}$ ), and the TWIP effect stems from deformation twinning. The TWIP effect provides a good combination of ductility and strength; therefore, these steels have drawn much attention as automobile materials. Fe-30Mn-6Si and Fe-30Mn-3Si-3Al (mass\%) alloys have the typical compositions for shape memory alloys (SMAs) and TWIP steels, respectively. The compositions show a similarity; the Si content is substituted by $\mathrm{Al}$ in the SMA, which is the composition of the TWIP steel. In the previous work, ${ }^{6}$ ) we reported that an Fe-30Mn$5 \mathrm{Si}-1 \mathrm{Al}$ alloy that had a similar composition to that of the SMA and TWIP steel showed a good shape memory and mechanical properties due to the coexistence of $\varepsilon$-martensite and deformation twins.

There are three deformation modes in FCC alloys with low stacking fault energy; $\varepsilon$-martensitic transformation, deformation twinning and slip deformation. All the deformation modes are attributed to the motions of the Shockley partial dislocations. Intrinsic stacking faults are formed on every $\{111\}$ plane, producing a twinning structure, while the $\varepsilon$ martensite is accompanied by the formation of stacking faults on alternate $\{111\}$ planes. The surface traces of $\varepsilon$-martensite and deformation twins have specific surface relief angles due to the periodic overlapping of stacking faults. In addition, the deformation products of the FCC alloys have the morphology of a plate-like structure. The identification of $\varepsilon$-martensite and deformation twins by comparing the experimental and theoretical values of relief angles has been reported as

\footnotetext{
*1 This Paper was Originally Published in Japanese in J. Japan Inst. Metals 73 (2009) 174-179.

${ }^{* 2}$ Graduate Student, Shibaura Institute of Technology. Present address: University of Tsukuba, Tsukuba 305-0047, Japan
}

well. ${ }^{7,8)}$ We examined the deformation microstructure of an $\mathrm{Fe}-30 \mathrm{Mn}-5 \mathrm{Si}-1 \mathrm{Al}$ alloy by atomic force microscope (AFM) according to the described method, ${ }^{7,8)}$ and confirmed that the deformation twins and the $\varepsilon$-martensite formed within the alloy. ${ }^{9)}$ In this work, we report deformation microstructures at various deformation stages, and the transition of deformation modes. The mechanisms associated with the $\varepsilon$-martensitic transformation, twinning and slip deformation will be discussed based on the results.

\section{Experimental}

\subsection{Deformation microstructure}

The Fe-30Mn-5Si-1Al (mass\%) alloy was prepared by vacuum induction melting. Hereafter, the compositions will be expressed as mass\%. Hot rolling, forging at $1273 \mathrm{~K}$ and subsequent solution treatment at $1273 \mathrm{~K}$ for $3 \mathrm{~h}$ were performed. The samples with dimensions of $4.0 \mathrm{~mm} \times$ $1.0 \mathrm{~mm} \times 30.0 \mathrm{~mm}$ were cut with spark erosion. Mechanical and electrolytic polishing were performed to obtain a clean surface for AFM observations. Tensile deformations were made at a strain rate of $1.7 \times 10^{-4}\left(\mathrm{~s}^{-1}\right)$ at room temperature. The samples were deformed to $200 \mathrm{MPa}, 300 \mathrm{MPa}$, and $400 \mathrm{MPa}$, and then AFM observations were conducted on the surfaces of the tensiled samples. Figure 1 shows a typical stress-strain curve of the alloy. The stresses of $200 \mathrm{MPa}$, $300 \mathrm{MPa}$, and $400 \mathrm{MPa}$ correspond to the adjacent yield stress and sufficient plastic deformed conditions. The plastic strains after deformation to $200 \mathrm{MPa}, 300 \mathrm{MPa}$, and $400 \mathrm{MPa}$ were $0.2 \%, 2.1 \%$, and $7.4 \%$, respectively.

\subsection{Surface relief analysis}

As described, both the twinning and the $\varepsilon$-martensite arise from the motions of Shockley partial dislocations on $\{111\}_{\gamma}\langle 112\rangle$, and produce surface reliefs. Hereafter, the three dimensional Miller index will be for the FCC structure. The relationships among the surface relief angle and the crystal orientations are given by ${ }^{7)}$ 


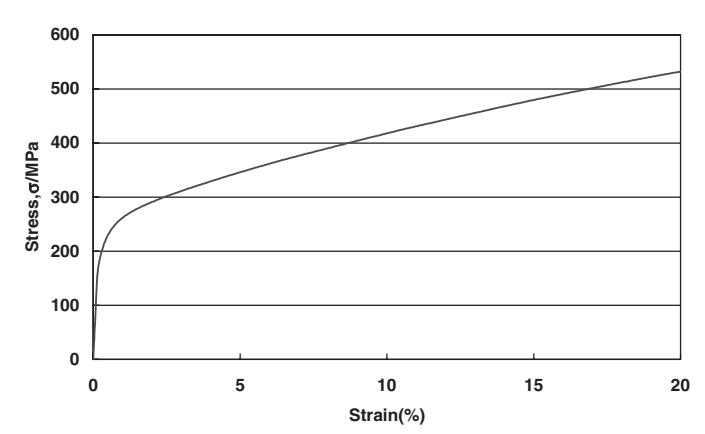

Fig. 1 Stress-strain curve for the Fe-30Mn-5Si-1Al alloy.

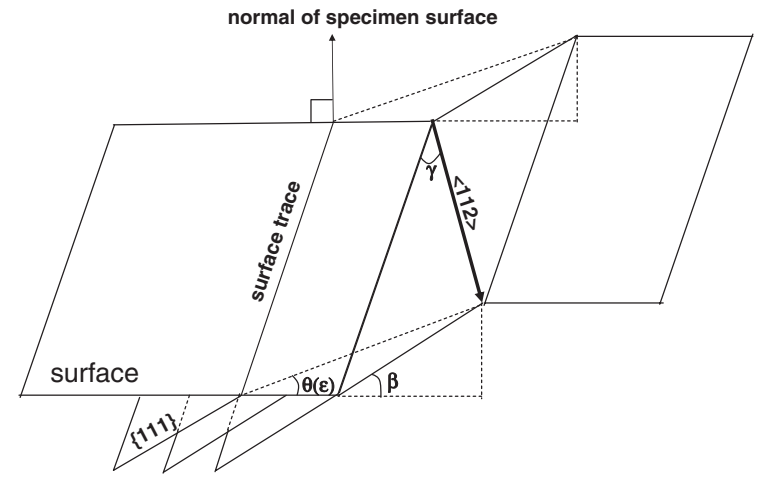

Fig. 2 Schematic illustration of the formation of a surface relief due to $\varepsilon$-martensitic transformation (In the case of deformation twins, the intermediate $\{111\}$ plane moves).
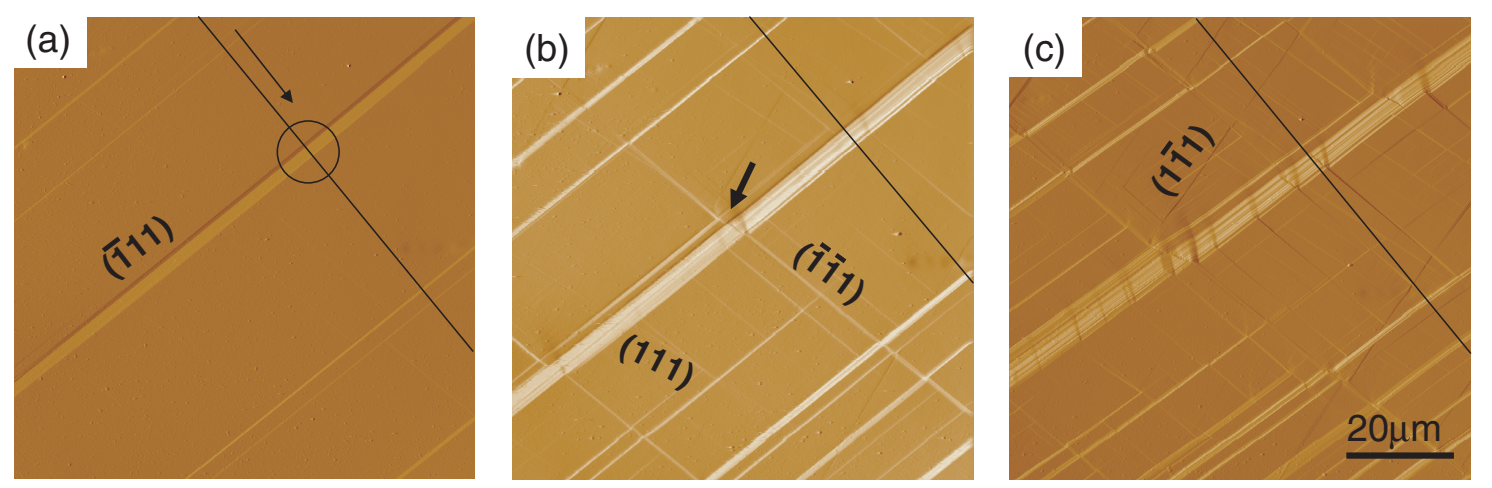

Fig. 3 Atomic force micrographs of the Fe-30Mn-5Si-1 Al alloy: images were taken after the tensile deformation at (a) $0.2 \%$, (b) $2.1 \%$, and (c) $7.4 \%$.

$$
\begin{aligned}
& \tan \theta(T)=\left(\sin \gamma \sin ^{2} \beta\right) /(\sqrt{2}+\sin \gamma \cos \beta \sin \beta) \\
& \tan \theta(\varepsilon)=\left(\sin \gamma \sin ^{2} \beta\right) /(2 \sqrt{2}+\sin \gamma \cos \beta \sin \beta)
\end{aligned}
$$

where $\beta$ and $\gamma$ are the angles between the surface and the $\{111\}$ plane and between the Burgers vector and the surface trace, respectively. A schematic illustration of the geometric relationship for the twinning and the $\varepsilon$-martensitic transformation is shown in Fig. 2. The surface crystal orientation, $\beta$ and $\gamma$ are determined from the angles obtained from the four $\{111\}$ traces. $^{10)}$ The theoretical angles for 12 kinds of $\{111\}\langle 112\rangle$ shear directions were calculated from eqs. (1) and (2). We have identified the deformation products through the comparisons between the theoretical and experimental values of the angles. In this work, we have observed the grains where the four kinds of surface traces appeared to determine the surface crystal orientation using AFM.

\section{Results and Discussion}

\subsection{Continuous transition of deformation modes}

Figures 3(a) to (c) show the AFM micrographs of the samples deformed by the $0.2 \%, 2.1 \%$ and $7.4 \%$ strain, respectively. The tensile direction is the horizontal direction. The micrographs were obtained from the same region of the grain. The differences among the contrasts indicate the differences in the surface relief angles. In this work, we focused on the deformation products described by the wide white contrast (hereafter, the deformation products are denoted by Relief X). The light brown contrast shows the FCC parent phase. The surface crystal orientation was determined to be [128] from the four surface traces shown in Fig. 3(b), where Relief $X$ is on the $(\overline{1} 11)$ plane. Table 1 shows the calculated $\beta, \gamma$ and surface relief angles of $\theta(\varepsilon)$ and $\theta(\mathrm{T})$ for the 12 kinds of $\mathrm{a} / 6\langle 112\rangle$ shear.

The relief angle of Relief $X$ in Fig. 3(a) is $10.5^{\circ}$. The influence of the surface tilt of the parent phase is removed by adding or subtracting the tilt angle from a measured relief angle. Thus, the relief angle does not include the surface tilt angle of the parent phase caused by the rotation of the grain and the specimen. The relief angle has a good agreement with the calculated angle for $\varepsilon$-martensite shown in Table 1, indicating that Relief $\mathrm{X}$ is a $\varepsilon$-martensite. The positive values of $\gamma, \theta(\varepsilon)$ and $\theta(\mathrm{T})$ corresponding to the [11 2 ] shear direction show a slope tilted towards the upper right direction in the AFM image. Relief $X$ has a positive slope shown using a lighter color than that of the parent phase. On the other hand, the calculated values for the $[\overline{2} \overline{1} \overline{1}]$ and [12 $\overline{1}]$ shear direction have negative values. The dark brown contrast along the left side of Relief $\mathrm{X}$ was obtained to be $-10.1^{\circ}$, which corresponds to the calculated value of the $\varepsilon$-martensite with the [ $\overline{2} \overline{1} \overline{1}]$ shear direction. This $\varepsilon$-martensite with the [ $\overline{2} \overline{1} \overline{1}]$ shear direction cannot be induced by the tensile stress. However, it can be considered to be induced by the local compressive stress in the grain. The local compressive stress field may arise from the constraint of the grain, existences of deformation products or influences from other grains. 
Table 1 Theoretical values of the surface relief angle on (1111) (in degrees).

\begin{tabular}{|c|c|c|c|}
\hline (111) & {$[11 \overline{2}]$} & {$[1 \overline{2} 1]$} & {$[\overline{2} 11]$} \\
\hline$\beta$ & 40.4 & 40.4 & 40.4 \\
\hline$\gamma$ & -83.1 & 23.1 & 143.1 \\
\hline$\theta(\mathrm{T})$ & -24.3 & 5.9 & 8.4 \\
\hline$\theta(\varepsilon)$ & -10.1 & 3.1 & 4.6 \\
\hline (i111) & 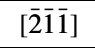 & {$[12 \overline{1}]$} & [1112] \\
\hline$\beta$ & 51.6 & 51.6 & 51.6 \\
\hline$\gamma$ & -131.4 & -11.4 & 108.6 \\
\hline$\theta(\mathrm{T})$ & -23.7 & -5.3 & 17.3 \\
\hline$\theta(\varepsilon)$ & -10.6 & -2.5 & 10.1 \\
\hline$(\overline{1} \overline{1} 1)$ & [2ī1] & [1121] & {$[\overline{1} \overline{1} \overline{2}]$} \\
\hline$\beta$ & -69.3 & -69.3 & -69.3 \\
\hline$\gamma$ & 154.8 & 34.8 & -85.2 \\
\hline$\theta(\mathrm{T})$ & 16.3 & 22.2 & -26.6 \\
\hline$\theta(\varepsilon)$ & 7.9 & 10.7 & -15.4 \\
\hline$(1 \overline{1} 1)$ & {$[21 \overline{1}]$} & {$[\overline{1} \overline{2} \overline{1}]$} & [1112] \\
\hline$\beta$ & -60.4 & -60.4 & -60.4 \\
\hline$\gamma$ & -13.3 & -133.3 & 73.3 \\
\hline$\theta(\mathrm{T})$ & -6.6 & -17.7 & 35.8 \\
\hline$\theta(\varepsilon)$ & -3.4 & -9.9 & 16.7 \\
\hline
\end{tabular}

Namely, the formation of the compressive stress induced $\varepsilon$-martensite can be regarded as a result of a self-accommodation.

In Figs. 3(b) and (c), the width and the tilt angle of Relief $\mathrm{X}$ increase with tensile strain. Figure 4 corresponds to the circled area in the cross sectional profile of Fig. 3(a). The surface relief angle at the $0.2 \%$ tensile strain is $10.1^{\circ}$, which is a constant value corresponding to the $\varepsilon$-martensite. The relief angle and the width increase at the $2.1 \%$ tensile strain, producing four parts which have constant values of $9.2^{\circ}$, $16.7^{\circ}, 11.0^{\circ}$ and $17.4^{\circ}$. By referring to Table 1 , one can see that Relief $\mathrm{X}$ at the $2.1 \%$ tensile strain is composed of the $\varepsilon$-martensite and deformation twins on (111)[1리. The deformation twins appear within the $\varepsilon$-martensite plate generated at the $0.2 \%$ tensile strain, indicating that a continuous transition from a $\varepsilon$-martensite to the twins occurs during the deformation from the $0.2 \%$ to $2.1 \%$ tensile strain. The width of the compressive stress induced $\varepsilon$-martensite along Relief X shown in Fig. 3(a) obviously decreases with increasing tensile strain. $\varepsilon$-martensite has been known to transform reversely to the parent phase by heating. The same phenomena has also been reported to occur by stress in the opposite direction. ${ }^{11)}$ Therefore, the $\varepsilon$-martensite induced by local compressive stresses can be considered to transform reversely by a further tensile stress. A curved profile indicated by the arrows in Fig. 4 is attributed to a slip deformation, since it cannot be explained by the deformation twins and the $\varepsilon$-martensite. Although slip deformation in FCC crystals also occurs on $\{111\}$ planes, slip deformation is not associated with the regular overlapping of stacking faults. Namely, relief angles arising from slip deformation do not have a constant value. In addition, slip deformation can create a large shear by the multiplication of dislocations on the same $\{111\}$ plane, producing larger relief angles than

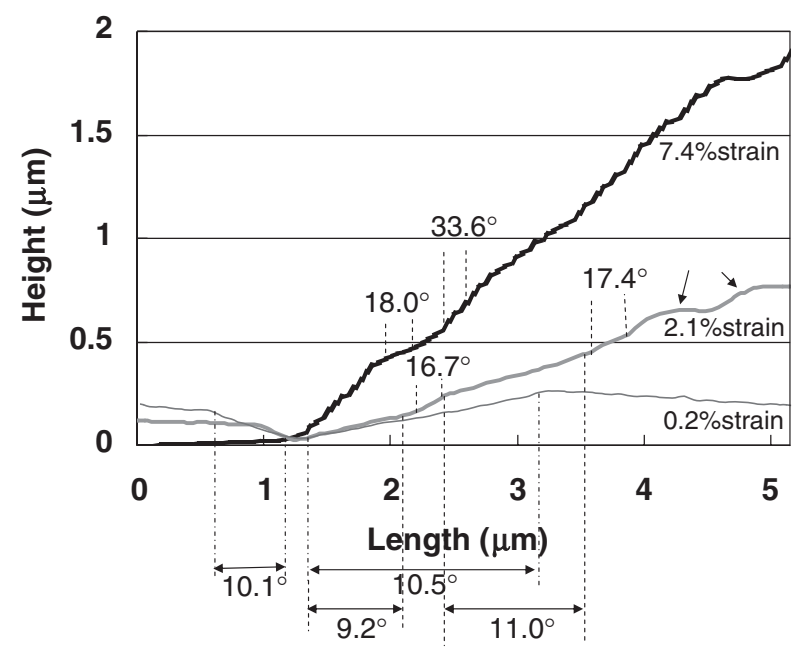

Fig. 4 Cross-sectional profiles corresponding to the black lines shown in Fig. 3(a) (c).

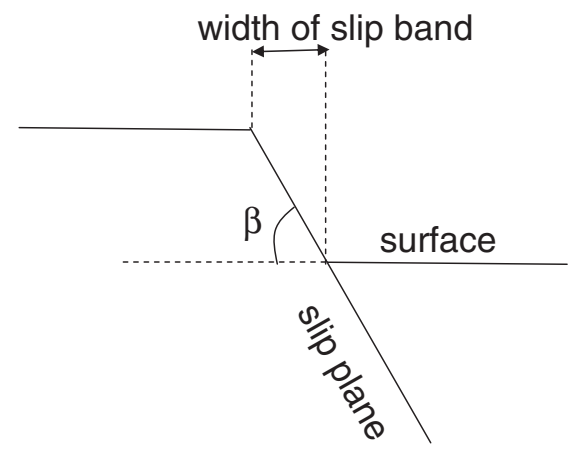

Fig. 5 Schematic illustration of a slip band. ${ }^{12)}$

that of the deformation twins. Thus, curved profiles and extremely large relief angles in Relief X stem from the slip deformation.

Curved reliefs become dominant at the $7.4 \%$ strain. The curved relief has an angle of $33.6^{\circ}$ as shown in Fig. 4. Other reliefs also have angles exceeding the $17.3^{\circ}$ theoretical angle for deformation twins, indicating that slip deformation occurs at the tensile strain. The maximum angle arising from the slip deformation is given by a model for slip bands, ${ }^{12)}$ as shown in Fig. 5. In the model, a surface relief is caused by the multiplication of dislocations on the same $\{111\}$ plane, producing a relief angle for slip deformation which is equal to $\beta$ as an angle between the $\{111\}$ plane and the surface. The maximum angle of reliefs along a $(\overline{1} 11)$ trace obtained by the mechanism in the grain $\left(\xi=13.5^{\circ}, \eta=7^{\circ}\right)$ is $51.6^{\circ}$. In Fig. 4 , the cross sectional profile corresponding to the $7.4 \%$ tensile strain has a curved shape and smaller angles than the maximum angle. This indicates that the surface relief is composed of multiple slip bands formed on some $\{111\}$ planes. The Burgers vector of the slip bands will be discussed in the next section. Notice that the relief angle of $18^{\circ}$ corresponding to the deformation twins still remains in the cross sectional profile at the $7.4 \%$ strain. The comparison between Figs. 3(b) and (c) shows that Relief X changes from a combination of deformation twins and $\varepsilon$-martensite to a 
combination of slip band and deformation twins with increasing strain from 2.1 to $7.4 \%$. We can conclude that a continuous transition of deformation modes occurs in the present $\mathrm{Fe}-30 \mathrm{Mn}-5 \mathrm{Si}-1 \mathrm{Al}$ alloy in which the deformation twins and the $\varepsilon$-martensite coexist: stress induced $\varepsilon$-martensitic transformation $\rightarrow$ twinning deformation $\rightarrow$ slip deformation.

\subsection{Intersection of $\varepsilon$-martensite}

Figure 6 shows a magnified AFM image of Fig. 3(b) ( $2.1 \%$ tensile strain). The two surface traces denoted by a and $\mathrm{b}$ that appear at the intersection of $(\overline{1} 11)$ and $(\overline{1} \overline{1} 1)$ traces are observed in this figure. The measured angle of the surface relief along the $(\overline{1} 11)$ trace is $6.9^{\circ}$, which identifies the relief as a $\varepsilon$-martensite. Although an intrusion of deformation twins in the (111) trace (Relief X) was observed, most parts of Relief $\mathrm{X}$ are occupied by the $\varepsilon$-martensite. Therefore, we assume that the intersection is composed of two different $\varepsilon$-martensite plates. The $\varepsilon$-martensite has the HCP structure and a specific crystallographic relationship with the parent phases: $\{111\}_{\gamma} / /(0001)_{\varepsilon}$. Hereafter, the four dimensional Miller indexes will be for the HCP. The primary slip plane of HCP crystals is (0001) which corresponds to the habit planes of the parent phase. Therefore, the traces a and $b$ are not attributed to the slip deformation within the $\varepsilon$-martensite plate. The $\alpha^{\prime}$-martensite ${ }^{13)}$ or a HCP twin $^{14)}$ has been reported

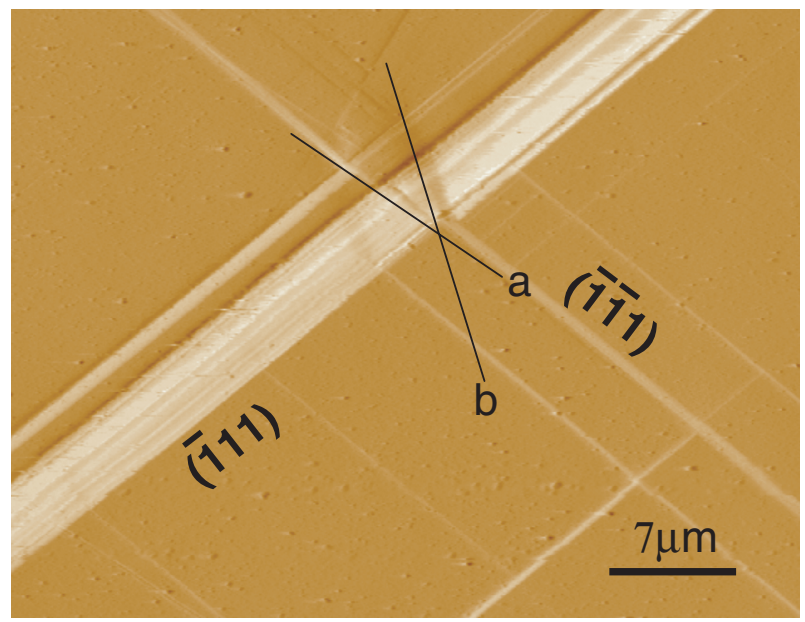

Fig. 6 Magnified image of the crossover site of Fig. 3(b). to form at the intersection of two $\varepsilon$-martensite plates. If the $\alpha^{\prime}$-martensite is induced by stress at room temperature, the $\alpha^{\prime}$-martensite can form at an intersection of $\varepsilon$-martensite plates. Since the stress induced $\alpha^{\prime}$-martensite does not occur in the Fe-30Mn-5Si-1 Al alloy at room temperature, a HCP twin may form at the intersection. Matsumoto et al. reported that $\{10 \overline{1} 1\}$ and $\{10 \overline{1} 2\}$ twins form at an intersection of the $\varepsilon$-martensite. ${ }^{14)}$ There are 6 equivalent planes for twinning planes: (10 $\overline{1} 1),(0 \overline{1} 11),(\overline{1} 101),(10 \overline{1} 2),(0 \overline{1} 12)$, and $(\overline{1} 102)$. Table 1 shows that the calculated angles between two surface traces along a $\{111\}$ plane and a HCP twinning plane are based on the Shoji-Nishiyama relation: $\left.{ }^{15}\right)[1 \overline{1} 00]_{\varepsilon} / /[\overline{2} \overline{1} \overline{1}]_{\gamma}$. Trace a shown in Fig. 6 produces a $75^{\circ}$ angle with the (111) trace and a $5^{\circ}$ angle with the (1) 11$)$ trace. Trace $b$ and the (111) trace or the ( $\overline{1} 11)$ trace produces an angle of $73^{\circ}$ or $29^{\circ}$, respectively. Traces a and b were identified as (0111) and (1011), respectively, from Table 2 . Although the (111) trace partially contains deformation twins which cause $\varepsilon$ martensite/twin interactions, its influence may be negligible due to the relatively-narrow region. Therefore, traces $a$ and $b$ at the interaction observed with AFM can be explained by the formation of two different $\{10 \overline{1} 1\}$ HCP twins. The formation of HCP twins may act as a self-accommodation to relax the stress concentration.

The Burgers vector for the slip band shown in Fig. 3(c) can be determined from the microstructure of the intersection in Fig. 6. The slip band can form by slip deformation on $(0001)_{\varepsilon}$ or $\{111\}_{\gamma}$. Kajiwara et al. investigated the microstructure within a $\varepsilon$-martensite plate by TEM and reported that the $\varepsilon$-martensite plates have the $\gamma / \varepsilon$ nano-lamella structure observed with TEM. ${ }^{16)}$ A slip deformation on $\{111\}_{\gamma}$ of

Table 2 Theoretical angle between the HCP-Twin trace and the $\{111\}$ trace (in degrees).

\begin{tabular}{ccc}
\hline FCC $\{111\}$ & $(\overline{1} 11)$ & $(\overline{1} \overline{1} 1)$ \\
\hline$(10 \overline{1} 1)$ & 75 & 26 \\
\hline$(0 \overline{1} 11)$ & 87 & 7 \\
\hline$(\overline{1} 101)$ & 12 & 67 \\
\hline$(10 \overline{1} 2)$ & 55 & 45 \\
\hline$(0 \overline{1} 12)$ & 58 & 22 \\
\hline$(\overline{1} 102)$ & 10 & 69 \\
\hline
\end{tabular}
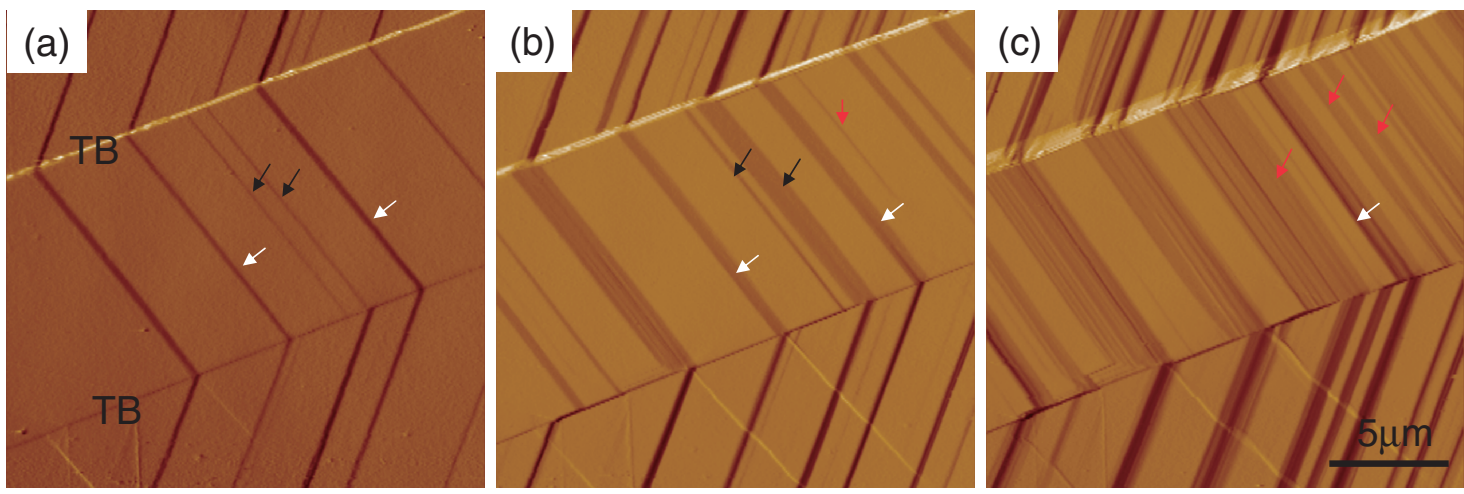

Fig. 7 Atomic force micrographs from a different grain in Fig. 3: images were taken after tensile deformation at (a) $0.2 \%$, (b) $2.1 \%$, and (c) $7.4 \%$. 
residual $\gamma$ in the $\gamma / \varepsilon$ lamella structure can produce the slip band. In addition, if the trailing partial dislocations which constitute the twins and the $\varepsilon$-martensite move on the same glide plane as the leading partial dislocations, it results in the same condition that occurs with a slip deformation of a FCC crystal. FCC and HCP slips can not be distinguished by surface observations due to the same slip direction and size of the Burgers vectors. Assuming that the FCC slip deformation occurs on the $(\overline{1} 11)$ plane, the possible slip directions are

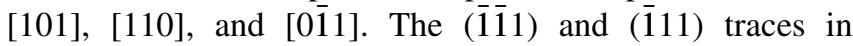
Figs. 3(b) and (c) recede each other with increasing strain. This indicates that the slip system is $(\overline{1} 11)$ [0 $\overline{1} 1]$. The slip system of a HCP crystal corresponding to the FCC slip system is (0001)[1 $\overline{2} 10]$.

\subsection{Correspondence with shape memory effects}

We reported that the present Fe-30Mn-5Si-1Al alloy has a shape memory effect. ${ }^{17)}$ The shape recovery strain was $1.8 \%$ with a $4 \%$ pre-strain. This value is smaller than that of conventional ferrous SMAs; an Fe-28Mn-6Si-5Cr alloy has a $2.1 \%$ recovery strain. However, the Fe-30Mn-5Si-1 Al alloy showed a $2.2 \%$ recovery strain when a $8 \%$ pre-strain was applied. Thus, the Fe-30Mn-5Si-1Al alloy was revealed to have a good maximum recovery strain with approximately a $8 \%$ pre-strain. Even though the analysis in Fig. 3(c) has shown that slip deformation becomes dominant at a $7.4 \%$ strain, the pre-strained sample showed a good shape memory effect by heating. Therefore, numerous stress-induced $\varepsilon$ martensite plates still form at the $7.4 \%$ tensile strain and provide the shape memory effect.

Typical AFM micrographs at different deformation stages are shown in Figs. 7(a) to (c) to confirm the formation of the $\varepsilon$-martensite at the $7.4 \%$ strain. The traces denoted by the TB are the annealing twin boundaries. The four traces observed in the lower left part of Fig. 7(a) determine the crystal orientation of the parent phase; the surface orientations of $\xi=38^{\circ}$ and $\eta=-10^{\circ}$ were obtained. The Miller indexes and some theoretical values for the deformation twins were calculated using the symmetric orientation relationship of the twins. The annealing twin boundaries are on $(\overline{1} \overline{1})$. Notice the $(1 \overline{1} 1)$ traces across the twin boundaries in Fig. 7(a). The possible shear directions for the twinning and $\varepsilon$-martensitic transformation are [ $\overline{2} \overline{1} 1]$ and $[1 \overline{1} \overline{2}]$. The theoretical angles for the twinning and $\varepsilon$ martensitic transformation are $\theta(\varepsilon)=3.2^{\circ}$, and $\theta\left(\gamma_{\mathrm{T}}\right)=6.4^{\circ}$ in the [ $\overline{2} \overline{1} 1]$ shear direction, and $\theta(\varepsilon)=14.5^{\circ}$, and $\theta\left(\gamma_{\mathrm{T}}\right)=$ $26.5^{\circ}$ in the [112] shear direction. The tilt angles of the surface reliefs represented by the white arrows in Fig. 7(a) were $3.3^{\circ}$ and $3.9^{\circ}$ respectively, indicating that both are $\varepsilon$ martensite formed by the [2 $\overline{1} 1]$ shear. The surface reliefs indicated by the black arrows have angles of $1.8^{\circ}$ and $2.3^{\circ}$. They are obviously lower than all the theoretical values shown in Table 1 . The smaller angles may be caused by the formation of a fine stacking structure in [2 $\overline{1} 1] \varepsilon$-martensite such as the $\varepsilon / \gamma$ nano-lamella structure reported in Fe-Mn-Si based SMAs. ${ }^{16)}$

The angles of the surface reliefs represented by the white arrows increased to $5.9^{\circ}$ and $5.7^{\circ}$ in Fig. 7(b). One of the reliefs marked by the black arrow has an angle of $5.3^{\circ}$. These values correspond quite well to the theoretical value of the deformation twins. In addition, the other one indicated by the black arrow has an angle of $3.1^{\circ}$ which corresponds to the $\varepsilon$ martensite. As a result, the $\varepsilon$-martensite and the $\gamma / \varepsilon$ lamella were found to change to deformation twins and $\varepsilon$-martensite, respectively. However, the surface reliefs identified as the deformation twins showed a slightly-lower tilt angle than the theoretical one, implying that the deformation twins have lamella structures including residual $\gamma$ and $\varepsilon$. A new trace with a $3.4^{\circ}$ tilt angle was also observed to appear at the $2.1 \%$ strain at the parts marked by the red arrows.

The width and the tilt angles of the surface reliefs indicated by the white and black arrows, respectively, increase in Fig. 7(c). For example, the part represented by the white arrow has a tilt angle of $6.5^{\circ}$ corresponding to the deformation twins. Thus, most surface reliefs are composed of twins and slip bands. On the other hand, new $\varepsilon$-martensite plates were observed at the parts marked by the three red arrows; the tilt angles were $3.8^{\circ}, 3.4^{\circ}$ and $3.7^{\circ}$, respectively.

Consequently, the transition of deformation modes, $\varepsilon$ martensite $\rightarrow$ deformation twin $\rightarrow$ slip band, was found to be the common phenomenon in several different grains. Intermediate relief angles associated with the fine lamella structure were also observed. Moreover, the $\varepsilon$-martensite plate in the grain shown in Fig. 3 changed to a slip band with increasing tensile strain from $0.2 \%$ to $7.4 \%$. Although the slip band and deformation twins did not contribute to the shape memory properties, new $\varepsilon$-martensite plates appeared in the residual $\gamma$ phase. We consider that the $\varepsilon$-martensite plates that formed at a large deformation stage support the good shape memory property of the present alloy with a $8 \%$ pre-strain.

\section{Conclusion}

We have investigated the deformation modes at various plastic deformation stages in the Fe-30Mn-5Si-1Al alloy where deformation twins and $\varepsilon$-martensite coexist. The following results were obtained with AFM observations.

(1) Continuous transitions of deformation products occur at the same region with increasing tensile strain: parent phase $(\gamma) \rightarrow$ stress induced $\varepsilon$-martensite $\rightarrow$ deformation twinning $(\gamma) \rightarrow$ slip bands in the deformation twins.

(2) Two different $\{10 \overline{1} 1\}$ HCP twins form at the intersection of $\varepsilon$-martensite plates formed on different $\{111\}$ habit planes.

(3) Although $\varepsilon$-martensite plates that formed at a small strain change continuously to deformation twins and slip bands with increasing tensile strain, new $\varepsilon^{-}$ martensite plates form from the residual $\gamma$ phases and provide a good shape memory effect for the alloy.

\section{Acknowledgement}

This work was supported financially by the New Energy and Industrial Technology Development Organization (NEDO) and the Grant-in-Aid for Scientific Research Fund (C) from the Japan Society for the Promotion of Science (JSPS). We thank the Materials Manufacturing and Engineering Station at the National Institute for Materials Science (NIMS) for the materials processing. 


\section{REFERENCES}

1) A. Sato, E. Chishima, K. Soma and T. Mori: Acta Metall. 30 (1982) 1177-1183.

2) H. Otsuka, H. Yamada, T. Maruyama, H. Tanahashi, S. Matsuda and M. Murakami: ISIJ Int. 30 (1990) 674-679.

3) M. Murakami, H. Otsuka, G. Suzuki and M. Matsuda: Proc. ICOMAT, (1986) pp. 985-990.

4) O. Grassel and G. Frommeyer: Mater. Sci. Technol. 14 (1998) 1213 1217.

5) S. Vercammen, B. C. De Cooman, N. Akdut, B. Blanplain and P. Wollants: Steel Res. 74 (2003) 370-375.

6) M. Koyama, M. Murakami, K. Ogawa, T. Kikuchi and T. Sawaguchi: Mater. Trans. 48 (2007) 2729-2734.

7) N. Bergeon, S. Kajiwara and T. Kikuchi: Acta Mater. 48 (2000) 4053 4064.
8) D. Z. Liu, S. Kajiwara, T. Kikuchi and N. Shinya: Philos. Mag. 83 (2003) 2875-2897.

9) M. Koyama, M. Murakami, K. Ogawa, T. Kikuchi and T. Sawaguchi: Mater. Trans. 49 (2008) 812-816.

10) S. Takeuchi, T. Homma and S. Ikeda: J. Japan Inst. Metals 22 (1958) 320-323.

11) T. Sawaguchi, L. Bujoreanu, T. Kikuchi, K. Ogawa, M. Koyama and M. Murakami: Scr. Mater. 59 (2008) 826-829.

12) A. Ewing and W. Rosenhain: Phil. Trans. A 195 (1900) 279.

13) A. Sato, K. Soma and T. Mori: Acta Metall. 30 (1982) 1901-1907.

14) S. Matsumoto, A. Sato and T. Mori: Acta Metall. Mater. 42 (1994) 1207-1213.

15) Z. Nishiyama: Kinzoku no kenkyuu 11 (1934) 561.

16) S. Kajiwara and K. Ogawa: Mater. Trans., JIM 34 (1993) 1169-1176.

17) M. Koyama, M. Murakami, K. Ogawa, T. Kikuchi and T. Sawaguchi: Proc. SMST (2007) pp. 653-657. 